Article

\title{
Neuritogenic and Neuroprotective Effects of Polar Steroids from the Far East Starfishes Patiria pectinifera and Distolasterias nipon
}

\author{
Natalia V. Palyanova ${ }^{1}$, Tatyana M. Pankova ${ }^{1}$, Marina V. Starostina ${ }^{1}$, Alla A. Kicha ${ }^{2, *}$, \\ Natalia V. Ivanchina ${ }^{2}$ and Valentin A. Stonik ${ }^{2}$ \\ 1 Institute of Molecular Biology and Biophysics, SB RAMS, Novosibirsk 630117, Russia; \\ E-Mails: natasha@soramn.ru (N.V.P.); pan@soramn.ru (T.M.P.); marina@soramn.ru (M.V.S.) \\ 2 G.B. Elyakov Pacific Institute of Bioorganic Chemistry, FEB RAS, Vladivostok 690022, Russia; \\ E-Mails: ivanchina@piboc.dvo.ru (N.V.I.); stonik@piboc.dvo.ru (V.A.S.) \\ * Author to whom correspondence should be addressed; E-Mail: kicha@piboc.dvo.ru; \\ Tel.: +7-423-2312-360; Fax: +7-423-2314-050.
}

Received: 5 March 2013; in revised form: 18 April 2013 / Accepted: 22 April 2013 / Published: 3 May 2013

\begin{abstract}
The neuritogenic and neuroprotective activities of six starfish polar steroids, asterosaponin $\mathrm{P}_{1}, \quad(25 S)-5 \alpha$-cholestane-3 $\beta, 4 \beta, 6 \alpha, 7 \alpha, 8,15 \alpha, 16 \beta, 26$-octaol, and

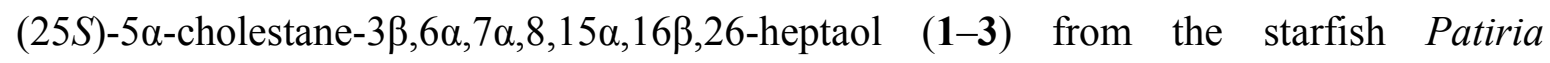
pectinifera and distolasterosides $\mathrm{D}_{1}-\mathrm{D}_{3}(\mathbf{4}-\mathbf{6})$ from the starfish Distolasterias nipon were analyzed using the mouse neuroblastoma (NB) C-1300 cell line and an organotypic rat hippocampal slice culture (OHSC). All of these compounds enhanced neurite outgrowth in NB cells. Dose-dependent responses to compounds 1-3 were observed within the concentration range of 10-100 nM, and dose-dependent responses to glycosides 4-6 were observed at concentrations of 1-50 nM. All the tested substances exhibited notable synergistic effects with trace amounts of nerve growth factor (NGF, $1 \mathrm{ng} / \mathrm{mL}$ ) or brain-derived neurotrophic factor (BDNF, $0.1 \mathrm{ng} / \mathrm{mL}$ ). Using NB cells and OHSCs, it was shown for the first time that starfish steroids 1-6 act as neuroprotectors against oxygen-glucose deprivation (OGD) by increasing the number of surviving cells. Altogether, these results suggest that neurotrophin-like neuritogenic and neuroprotective activities are most likely common properties of starfish polyhydroxysteroids and the related glycosides, although the magnitude of the effect depended on the particular compound structure.
\end{abstract}


Keywords: steroid; starfish; neuroblastoma; organotypic hippocampal slice culture; neuritogenesis; oxygen-glucose deprivation

\section{Introduction}

Neurotrophins, such as nerve growth factor (NGF) and brain-derived neurotrophic factor (BDNF), are essential for development, differentiation, survival and functional maintenance of neurons in the central and peripheral nervous systems [1,2]. Although neurotrophins are expected to have therapeutic potential in the treatment of neuronal injuries [3], they are rather unstable and too large to pass through the blood-brain barrier that limits their use in medicine. Therefore, low molecular weight compounds mimic the activity of neurotrophins and capable to cross blood-brain barrier should be interesting as promising therapeutic agents to treat traumatic or ischemic brain injuries and neurodegenerative diseases. In recent years, several low molecular weight substances from various natural sources have been shown to possess neurotrophic ability [4,5]. Some of the most effective compounds can be proposed as potential drugs.

Polyhydroxylated steroids and related steroid glycosides are the predominant secondary metabolites in starfish (the phylum Echinodermata, the class Asteroidea) [6-8]. Polyhydroxysteroids, as a rule, contain from four to nine hydroxyl groups in steroid nucleus and side chain. Related steroid glycosides have a polyhydroxylated steroid nucleus and one, two or rarely three monosaccharides units attached to polycyclic system, either to side chains or to steroid nucleus and side chain simultaneously. These compounds attract the attention not only because of their peculiar chemical structures but also due to the wide spectrum of their biological activities, including cytotoxic, antiviral, antibacterial, antibiofouling and antifungal effects [6-8]. Neuritogenic activity of steroid glycosides from the Okinawan blue starfish Linckia laevigata was revealed for the first time by Qi, Han and co-authors $[4,9,10]$ on pheochromocytoma PC12 cells. In our previous work, we demonstrated that polar steroids (polyhydroxysteroids and related glycosides) from various species of starfish could induce neurite outgrowth in mouse neuroblastoma C-1300 cell culture [11-13]. The present research is devoted to more detailed study of neurotrophic activities of six starfish steroid compounds: asterosaponin $\mathrm{P}_{1}$,

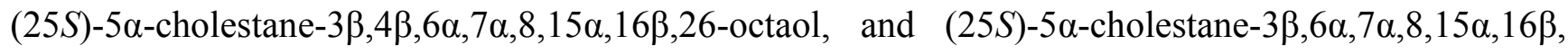
26-heptaol, designated as PP1, PP2, and PP3 (1-3), from Patiria (=Asterina) pectinifera [14], and distolasterosides $\mathrm{D}_{1}-\mathrm{D}_{3}$, designated as D1, D2, and D3 (4-6), from Distolasterias nipon [13] (Figure 1). Besides the investigation of neuritogenic activity of starfish steroids 1-6 at low concentrations, we analyzed their neuroprotective ability during oxygen-glucose deprivation using the mouse neuroblastoma C-1300 and organotypic rat hippocampal slice cultures. The starfishes $P$. pectinifera and D. nipon are common shallow waters species in the Northwestern Pacific. Compounds $\mathbf{1}-\mathbf{3}$ are the most abundant polar steroid constituents in P. pectinifera [14], whereas the substances 4-6 are the major polar steroid constituents in D. nipon [13]. Asterosaponin $\mathrm{P}_{1}$ represents the example of monoside with the 3-O-methyl-5- $O$-sulfonato- $\alpha$-L-arabinofuranose unit attached to $\mathrm{C}-24$ in a side chain while the both 2 and 3 are free nonsulfated polyhydroxylated compounds. Distolasterosides $\mathrm{D}_{1}-\mathrm{D}_{3}(\mathbf{4}-\mathbf{6})$ are the biosides, containing $\beta$-D-xylopyranose unit at C-3 and other monosaccharide residue at C-24 
( $\beta$-D-xylopyranose unit in $\mathbf{4}$ and $\mathbf{5}$, and $\beta$-D-glucopyranose unit in 6). Moreover, compound $\mathbf{5}$ have a double bond in the side chain at the position 22(23).

Figure 1. Structures of the compounds 1-6.

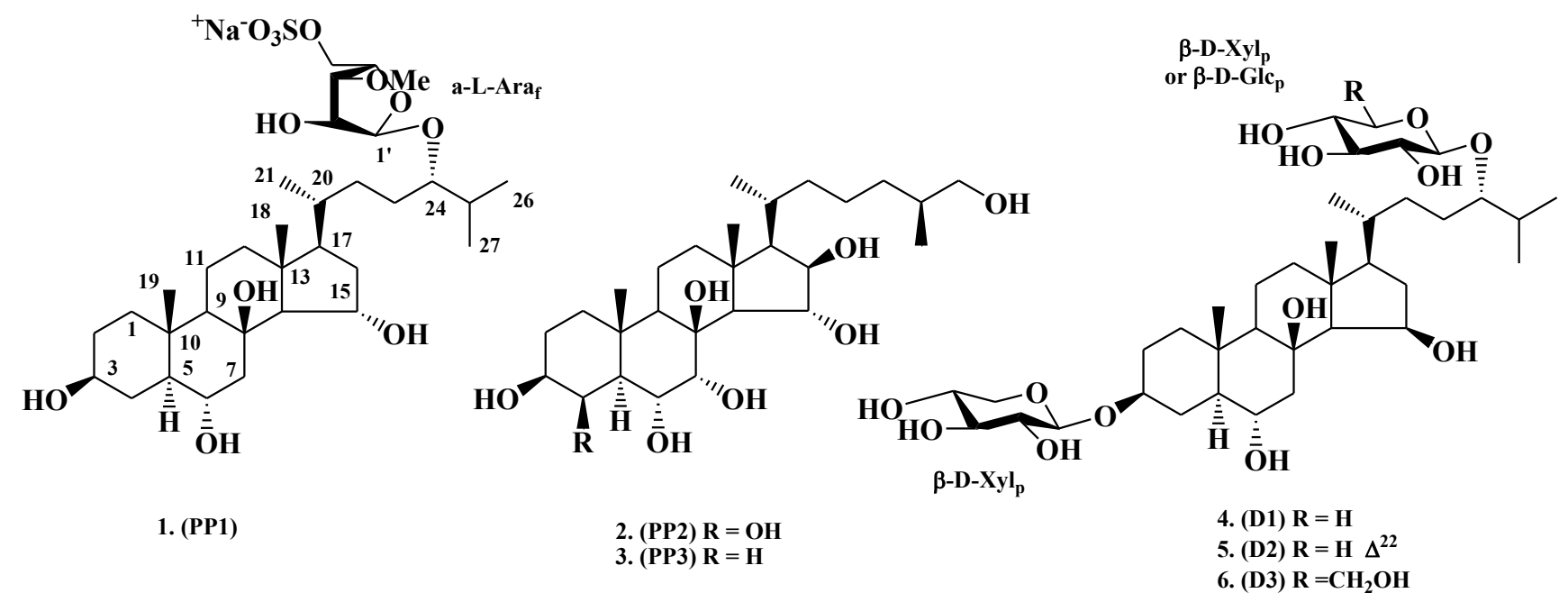

\section{Results and Discussion}

\subsection{Neuritogenic Activity of Starfish Polar Steroids in Cultured NB Cells}

Neuroblastoma (NB) cell cultures are widely used to study neuritogenic and neuroprotective properties of bioactive substances $[15,16]$. Usually there are several percent of spontaneously differentiating cells in NB C-1300 culture, and the number of differentiated cells increases after prolonged cultivation or cultivation in media with low serum content $[17,18]$. In our experiments, cells were maintained in 5\% bovine fetal serum. The number of cell passages did not exceed $8-10$, and each set of experiments contained a control to eliminate mistakes caused by the degree of spontaneous differentiation of the test culture. Thus, in our experiments from $14 \%$ to $25 \%$ of cells possessed morphological properties of mature neurons (Figures $2 \mathrm{~B}$ and $3 \mathrm{~B}$, controls). It was shown that dimethylsulfoxide (DMSO) also induced the differentiation of NB cells [19,20]. We did not observe significant differences in number of differentiated cells cultured with or without DMSO, possibly due to low final concentration of DMSO. Nevertheless, in all subsequent experiments DMSO was added to control cultures.

The neuritogenic activity of starfish polar steroids in living NB cells can be detected beginning at the second day of cultivation, as we have described previously [11-13]. Previous results have demonstrated that a series of polar steroids, including 1-3, manifested significant and nearly identical neuritogenic effects at doses of $2-20 \mu \mathrm{M}$, but these effects were slight or even absent at higher concentrations of approximately $40 \mu \mathrm{M}$ [11]. Polar steroids 4-6 from D. nipon were effective at lower concentrations $(0.1-2 \mu \mathrm{M})$ but demonstrated neurotoxic properties at higher concentrations $(20-40 \mu \mathrm{M})[13]$.

In the present work, we performed more detailed studies on the neuritogenic properties of compounds 1-6 at much lower concentrations within 10-100 nM for 1-3 and 1-50 nM for 4-6 in NB cells using silver-impregnated preparations. Our experiments demonstrated that compounds $\mathbf{1}$ and $\mathbf{3}$ 
increased the number of differentiated neurons (percentage of cells bearing neurites longer than two cell diameters or bearing more than two processes) after four days of incubation at concentrations of $50 \mathrm{nM}$ and higher. Compound 2 had the same effect at doses of $10 \mathrm{nM}$ and above, as did compounds 4-6 at doses of $5 \mathrm{nM}$ and above. Dose-dependent responses to all of the compounds were also observed after four days of incubation (Figures 2 and 3).

Figure 2. Neuritogenic activity of starfish steroids from $P$. pectinifera in NB C-1300 cells. (A) Images of control and steroid-treated cultures. Treatment with compounds PP1, PP2 or PP3 (1-3) at concentration of $100 \mathrm{nM}$ for 4 days; (B) Dose-dependent neuritogenic effects of compounds PP1, PP2, and PP3 (1-3). The data are presented as the mean values \pm SEM from representative experiments. $* p \leq 0.05$.

A
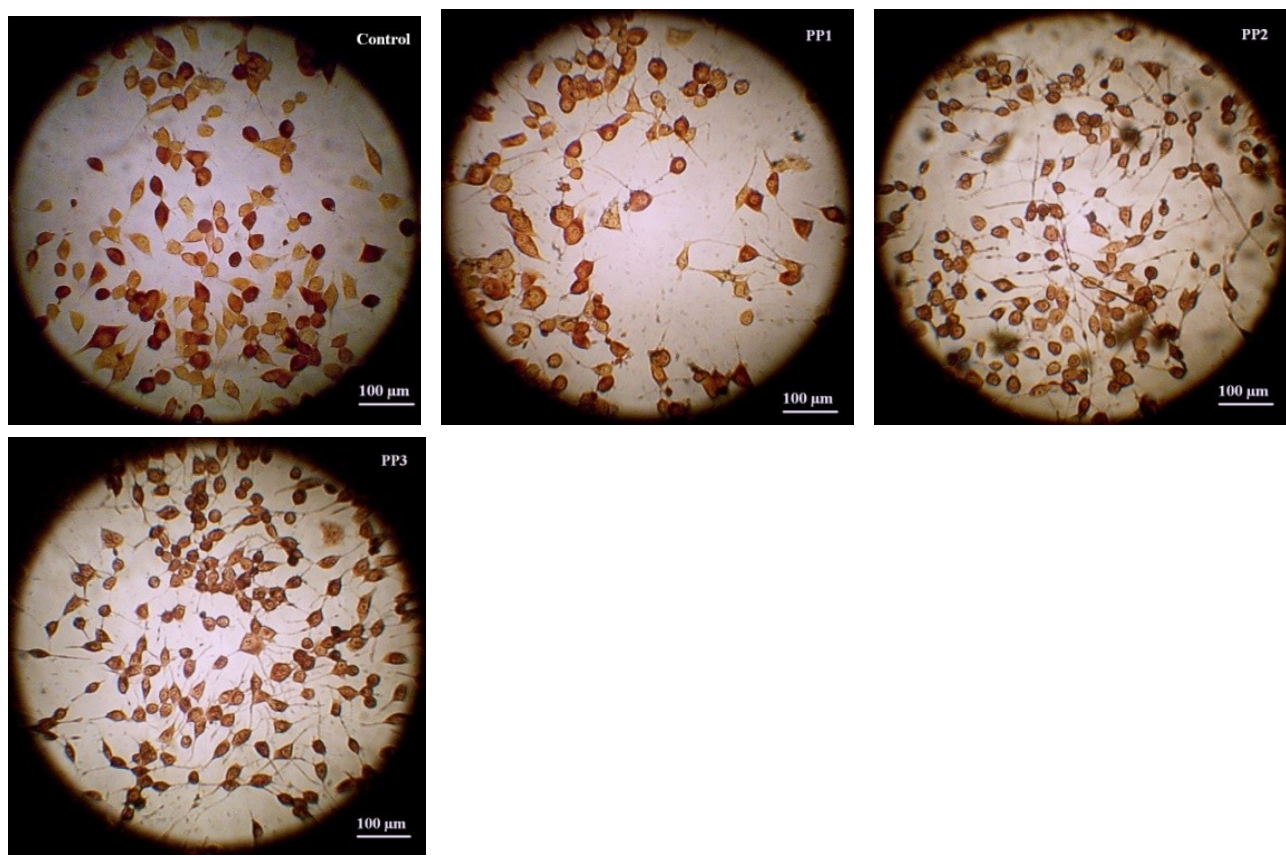

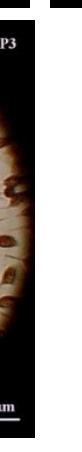

B

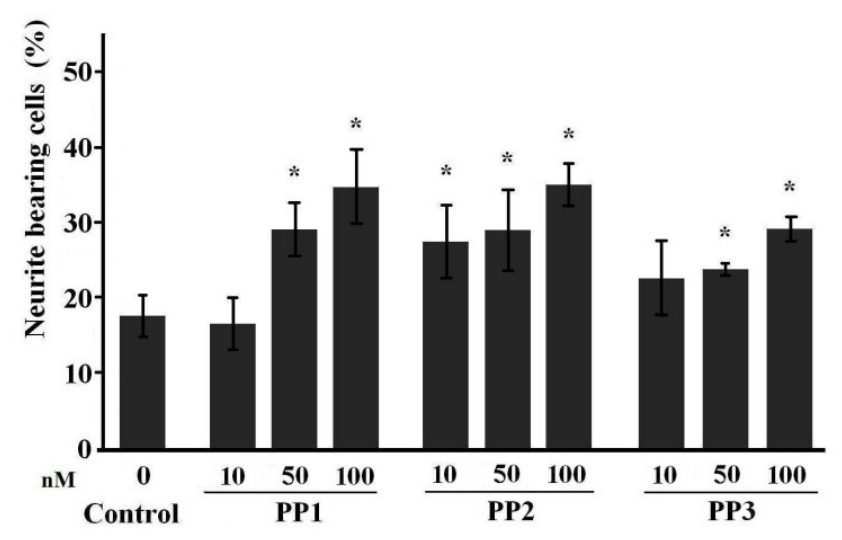


Figure 3. Neuritogenic activity of starfish steroids from D. nipon in NB C-1300 cells. (A) Images of control and steroid-treated cultures. Treatment with compounds D1, D2 or D3 (4-6) at concentration of $50 \mathrm{nM}$ for 4 days; (B) Dose-dependent neuritogenic effects of compounds D1, D2, and D3 (4-6). The data are presented as the mean values \pm SEM from representative experiments. ${ }^{*} p \leq 0.05$.

A
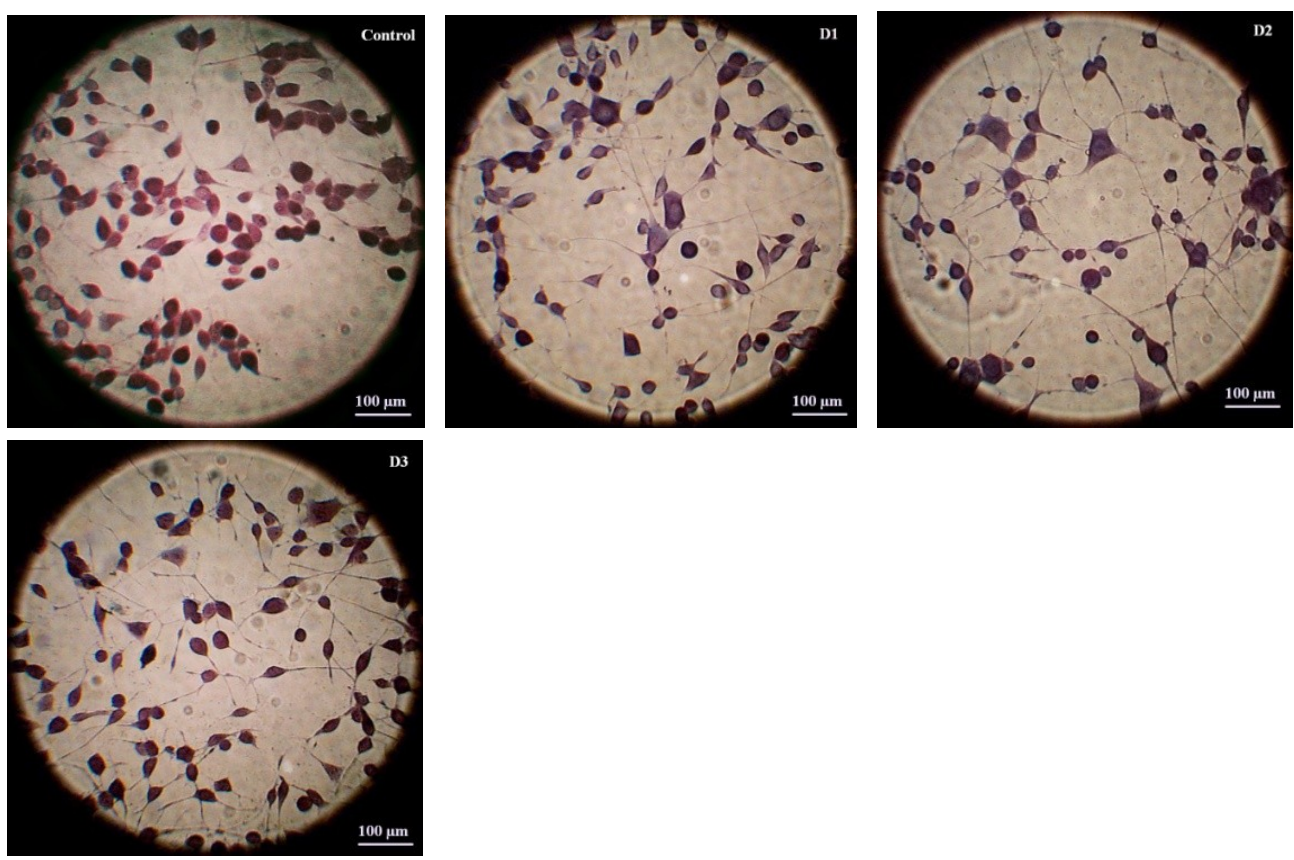

B

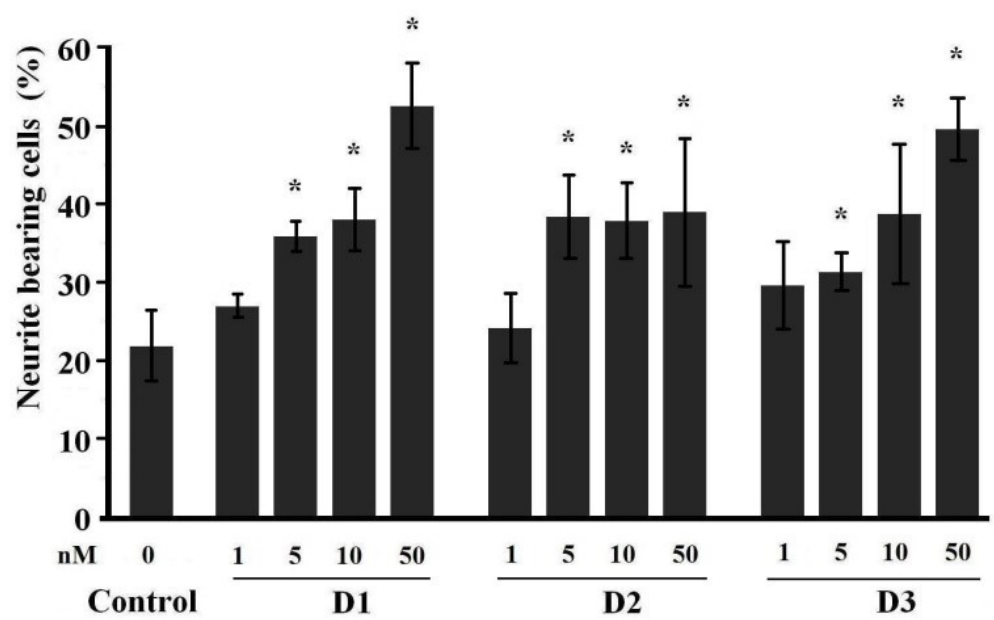

Culturing NB cells in the presence of the starfish steroid compounds also resulted in rapid primary neurite elongation. The mean process lengths \pm SEM in the NB cells cultivated with polar steroids 1-3 from P. pectinifera (at a concentration of $50 \mathrm{nM}$ ) were $91.56 \pm 5.5(\mathrm{PP} 1), 136 \pm 7.8$ (PP2), and $95.31 \pm 4.6(\mathrm{PP} 3) \mu \mathrm{m}$ compared to $105.4 \pm 4.3 \mu \mathrm{m}(\mathrm{NGF}, 10 \mathrm{ng} / \mathrm{mL})$ and $79.61 \pm 4.7 \mu \mathrm{m}$ in the controls. In the experiments using polar steroids 4-6 from D. nipon (at a concentration of $50 \mathrm{nM}$ ), the process lengths \pm SEM were $132.37 \pm 8.6$ (D1), $119.77 \pm 7.19$ (D2), and $113.18 \pm 7.1$ (D3) $\mu \mathrm{m}$ compared to $115.11 \pm 2.5 \mu \mathrm{m}(\mathrm{NGF}, 10 \mathrm{ng} / \mathrm{mL})$ and $103.94 \pm 5.7 \mu \mathrm{m}$ in the controls. Thus, the 
neuritogenic effects of the tested compounds in NB cell cultures were comparable to those of NGF. Compound 3 (PP3, $0.5 \mu \mathrm{M})$ significantly increased the mean number of processes per cell (2.4 \pm 0.2 times) compared to the control $(1.7 \pm 0.2$ times $)(p \leq 0.05)$; a similar effect was not observed in NB cells cultivated with the other tested steroids or NGF.

The ability to stimulate the differentiation of large neurons bearing several processes is a distinct feature of steroids 4-6 from D. nipon compared to other starfish steroids. Generally, the number of such neurons in self-differentiated NB C-1300 culture is insignificant (Figure 3A).

Qi, Han and co-authors have previously analyzed the biological action of a series of starfish steroid glycosides on pheochromocytoma PC-12 cells $[4,9,10]$. These cells are not capable of differentiation in the absence of neurotrophic stimuli and respond to NGF by switching from an immature chromaffin cell-like phenotype to a sympathetic neuron-like phenotype characterized the outgrowth of long neurites. In contrast, NB cells are capable of a spontaneous differentiation, and from $14 \%$ to $25 \%$ of cells were usually differentiated in our experiments. Additionally, NGF appears to stimulate neuritogenesis in only a portion of the neuroblast population in NB cultures. Thus, the differences in the properties of these cell lines underlie the discrepancy in the effective concentrations and the percentage of differentiated cells in our experiments versus the data obtained using PC-12 cells. However, the results obtained using the both cell models confirmed that starfish steroid glycosides and polyhydroxysteroids exhibit evident neuritogenic activities.

We also observed that the neuritogenic effects of starfish steroids on NB cells were synergistic with the effects of the neurotrophins NGF or BDNF. Both NGF and BDNF induced the differentiation of $\mathrm{NB}$ cells at concentrations of $10 \mathrm{ng} / \mathrm{mL}$; the percentage of neurite-bearing cells reached $46.32 \% \pm 5.79 \%$ in NGF-treated and $41.31 \% \pm 3.91 \%$ in BDNF-treated NB cells, while in the control, the mean value was $23.64 \% \pm 4.74 \%$. Ineffective (not inducing cell differentiation) concentrations were determined to be $1 \mathrm{ng} / \mathrm{mL}$ of NGF and $0.1 \mathrm{ng} / \mathrm{mL}$ BDNF. The simultaneous treatment of NB cultures with low concentrations of 1-6 and the ineffective concentrations of the neurotrophic factors significantly increased neuronal differentiation (Figure 4).

It is known that some endogenous steroids in mammals stimulate the synthesis of neurotrophins [21,22]. The synergistic effects between the starfish steroids and neurotrophins allow us to assume that the starfish steroids affect the expression of neurotrophins, such as mammalian steroids, or affect the signaling pathways activated by neurotrophins. In fact, it was previously established that the enhancement of NGF-induced neurite outgrowth by granulatoside A, a starfish steroid glycoside, in PC12 cells is attributable to both the increasing and maintenance of phosphorylation of the MAP kinase ERK1/2, although the upstream pathways are unclear [10]. However, it was reported in an article from our group on a related glycoside leviuscoloside $\mathrm{G}$ from the starfish Henricia leviucula that action of this compound on mouse skin JB6 $\mathrm{Cl} 41$ cells is rather complicated and includes the inhibition of ERKs, NF-kB and AP-1 activities [23]. We suggest that detailed mechanisms underlying the neuritogenic activities of steroid compounds from starfish including their action on all main MAP kinases and different nuclear factors should be investigated and have a plan to participate in these studies in the future. 
Figure 4. Synergistic effects of neurotrophins and starfish steroids on cell differentiation in NB C-1300 cells. (A,B) Effects of compounds from P. pectinifera and NGF (A) or BDNF (B). (C,D) Effects of compounds from D. nipon and NGF (C) or BDNF (D). The data are presented as the mean values \pm SEM from representative experiments. ${ }^{*} p \leq 0.05$.

A

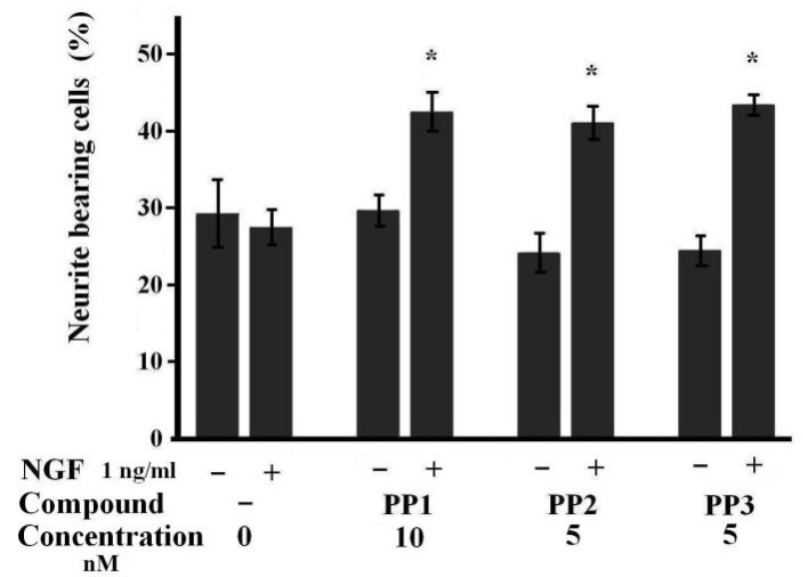

C

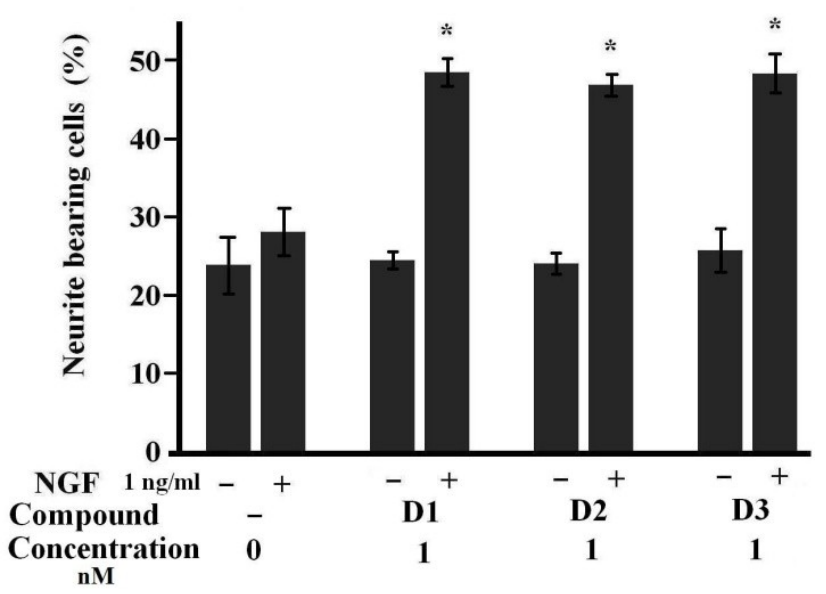

B

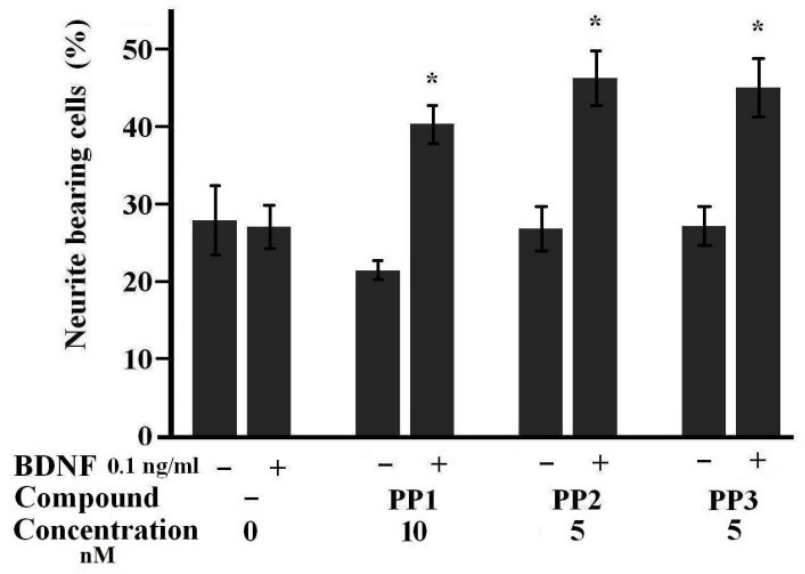

D

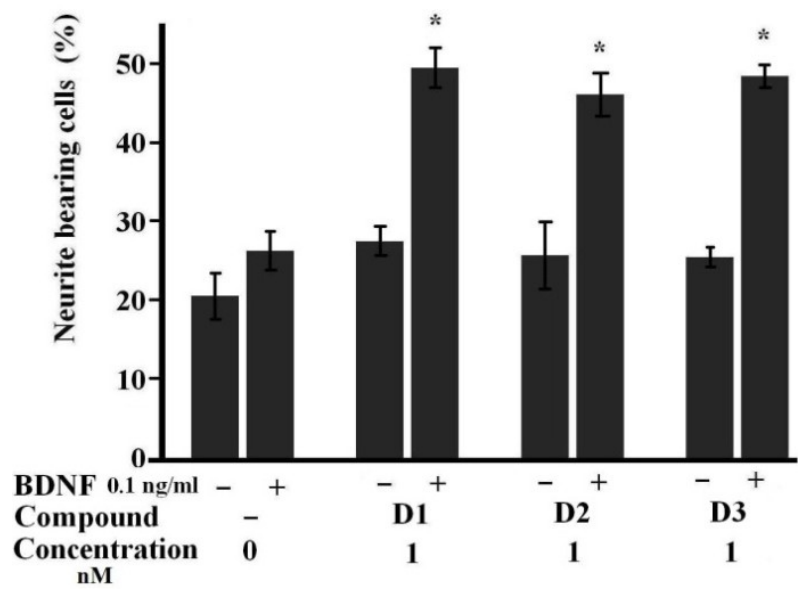

\subsection{Neuroprotective Activities of Starfish Polar Steroids in Oxygen-Glucose Deprivation Experiments} in Vitro

The neuroprotective effects of starfish polar steroids were assessed using NB C-1300 and organotypic hippocampal slice cultures. Both models are used in studies of the protective abilities of different drugs during oxygen-glucose deprivation (OGD) [15,24-26]. The OGD period appropriate for different neuroblastoma cell lines usually varied between $3 \mathrm{~h}$ up to $24 \mathrm{~h}$ depending on the cell line sensitivity [27,28]. Therefore, the time required for the most pronounced OGD effect in NB cells was determined in a preliminary set of experiments. We obtained reproducible results using NB C-1300 cell cultures that were exposed to OGD for $20 \mathrm{~h}$. In this case, the amount of surviving cells was approximately $14 \%-20 \%$, which was in good agreement with the data obtained using human neuroblastoma cell lines [27,29]. In control NB cultures without OGD treatment the percentage of living cells was approximately $85 \%-90 \%$. DMSO did not affect the cell surviving in control and OGD-treated cultures. 
Some differences in the protective abilities of steroids 1-3 from P. pectinifera and 4-6 from D. nipon were observed. Although protective effects were not observed for steroids $\mathbf{1}-\mathbf{3}$ from $P$. pectinifera at the concentration of $25 \mathrm{nM}$, reliable neuroprotective properties were detected for the same compounds at a concentration of $50 \mathrm{nM}$ (Figure 5A,B). However, the efficiency of protection at higher concentrations of these steroids, up to $2 \mu \mathrm{M}$, did not exceed the values established at a concentration of $50 \mathrm{nM}$. Steroids from D. nipon were more toxic and reduced the number of surviving cells in OGD-treated NB cells at concentrations of 0.5 or $2 \mu \mathrm{M}$ (data not shown). However, at concentration of $50 \mathrm{nM}$, these substances showed significant neuroprotective effects (Figure 5C).

Figure 5. Starfish steroids protect NB C-1300 cells from oxygen-glucose deprivation (OGD). Cultures were maintained in oxygen- and glucose-free medium for $20 \mathrm{~h}$, and the cell viability at $24 \mathrm{~h}$ after OGD was determined by counting the cells including trypan blue dye as the percentage of total cells. Starfish steroids were added twice to the cultures at the indicated concentrations for the OGD period and for $24 \mathrm{~h}$ after OGD. The data are presented as the mean values \pm SEM from representative experiments performed in triplicate. $* p<0.05$. (A) Compounds PP1, PP2, and PP3 (1-3) failed to increase cell viability at the concentration of $25 \mathrm{nM}$; (B) Protective effects of compounds PP1, PP2, and PP3 (1-3) at the concentration of $50 \mathrm{nM}$; (C) Protective effects of compounds D1, D2, and D3 (4-6) at the concentration of $50 \mathrm{nM}$.

A

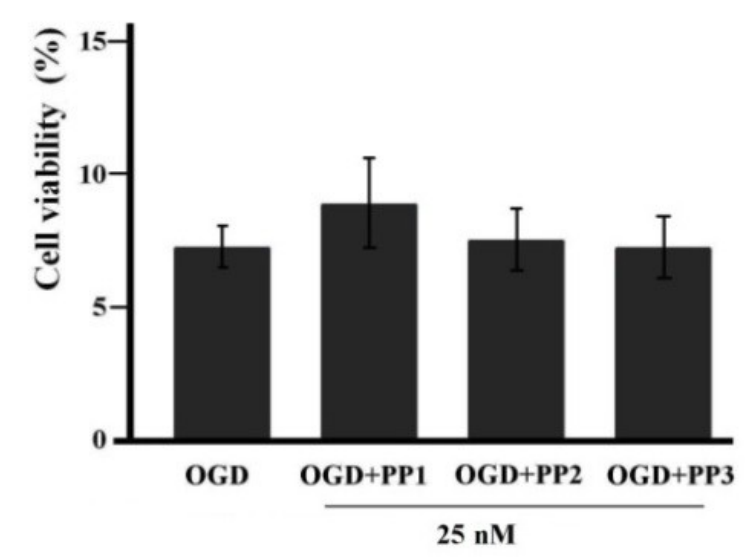

B

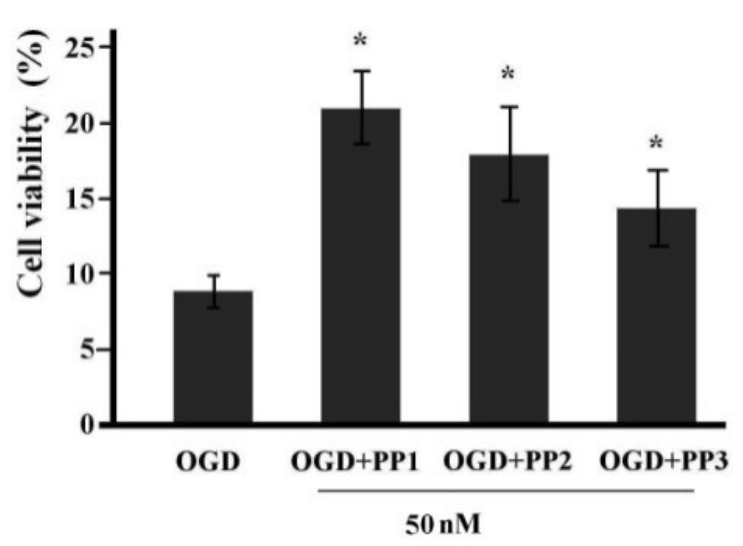

C

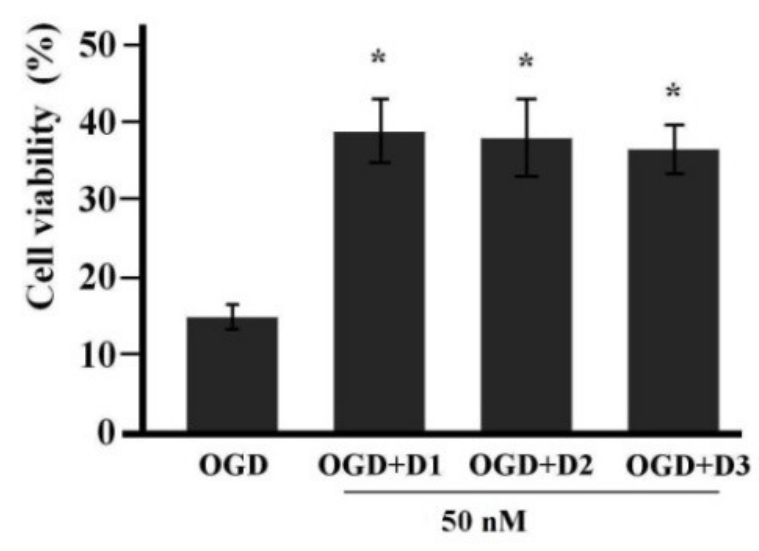


Recently, a hypoxia-inducible angiogenic peptide known as vascular endothelial growth factor (VEGF) was identified as a neuroprotector in studies on OGD in NB cell lines [27]. Interestingly, the neuroprotective effects of the polar starfish steroids were comparable to those elicited by VEGF. Possibly, the neuroprotective properties of the starfish steroids could result from their ability to induce cell differentiation. In several recent studies, the relationship between hypoxia and the stage of cell differentiation in solid NB tumors and NB cell lines has been established [30-32]. It was demonstrated that hypoxia provoked cell dedifferentiation by down-regulating genes associated with neuronal and upregulating genes associated with a neural crest-like phenotype [33,34], thus contributing to the malignancy of the tumor. Glucose deficiency potentiated the effect of hypoxia [35]. In turn, factors that stimulated NB cell differentiation showed protective activities against hypoxic injury [36].

Organotypic hippocampal slice cultures (OHSCs) demonstrate advantages compared with other organotypic systems used in vitro to study the protective effects of various substances because they maintain cell architecture, neuronal connections and electric activity, allowing for extended survival studies [37]. In terms of oxygen-glucose deprivation, OHSCs closely mimic the in vivo ischemia-induced injuries [25,26]. To study the neuroprotective activity in OHSCs we selected compounds PP1 (1), D1 (3) and D3 (6), because these compounds were some more effective in neuritogenic and neuroprotective assays on NB culture and were available for further studies. OHSC survival in the presence of $\mathbf{1}, \mathbf{3}$, and $\mathbf{6}(1 \mu \mathrm{M})$ was estimated visually during cultivation. After 7-8 days of cultivation, the OHSCs were fixed, stained with cresyl violet or impregnated with silver and studied under a light microscope. None of the tested compounds affected the development of hippocampal slices in culture or their adhesion to collagen in the absence of OGD. Slices maintained the normal arrangement of cellular layers and developed a growth area containing migrated fibroblasts and extended processes similar to control cultures. DMSO added to the culture medium in control OHSCs $(0.025 \%)$ did not affect the development of slices.

Only single dead cells were present in the control experiments when the OHSCs were stained with propidium iodide (Figure 6A), while massive cell death in various hippocampal areas and fascia dentata was observed in OHSCs exposed to OGD (Figure 6B). There was no significant difference in the amount of cell death OGD-treated and OGD + DMSO-treated cultures. That is in agreement with the previously reported data [26,38,39]. Compounds PP1 (1), D1 (4) and D3 (6) at a concentration of $1 \mu \mathrm{M}$ were tested in OHSCs under OGD conditions. These starfish steroids reduced the number of propidium-labeled dead cells (Figure 6A,B), providing evidence of neuroprotective properties similar to those of endogenous mammalian steroids previously analyzed using OGD-treated OHSCs [26,38]. The protective effects of mammalian steroids, in particular female sex hormones, have been widely reported in the different types of neuronal cells against various toxicities, including serum deprivation, oxidative stress, amyloid- $\beta$ peptide, and excitotoxicity [40]. The ability of estrogens to decrease ischemia/reperfusion injury was shown not only in OHSCs [26,38], but in animal models also [41,42]. Although estrogens are neuroprotective, the hormonal effects limit their clinical application, and the search of their analogues with neuroprotective function but lacking hormonal properties is actual. It was shown that the neuroprotective effects of sex hormones could result from their influence on the expression and/or function of neurotrophins [22,43].

Starfish polar steroids, namely, polyhydroxysteroids and related mono- and biglycosides, differ structurally from known protective steroids in mammals. Nevertheless, starfish steroids exhibit the 
similar neuroprotective abilities and their neuritogenic effects are synergic to neurotrophins. The molecular mechanisms of their effects remain to be investigated.

Figure 6. Effect of starfish steroids on cell damage induced by OGD in organotypic hippocampal slice cultures. (A) Representative confocal images of control slices and slices stained with propidium iodide (PI) $24 \mathrm{~h}$ after exposure to OGD. Starfish steroids were added to OHSCs for $24 \mathrm{~h}$ prior to and for $24 \mathrm{~h}$ after OGD; (B) Quantitative assay of cell damage $24 \mathrm{~h}$ after exposure to OGD. Five independent experiments were carried out. The data are presented as the mean values \pm SEM. * - a significant difference from exposure to $35 \min$ OGD, $p \leq 0.05$.

A
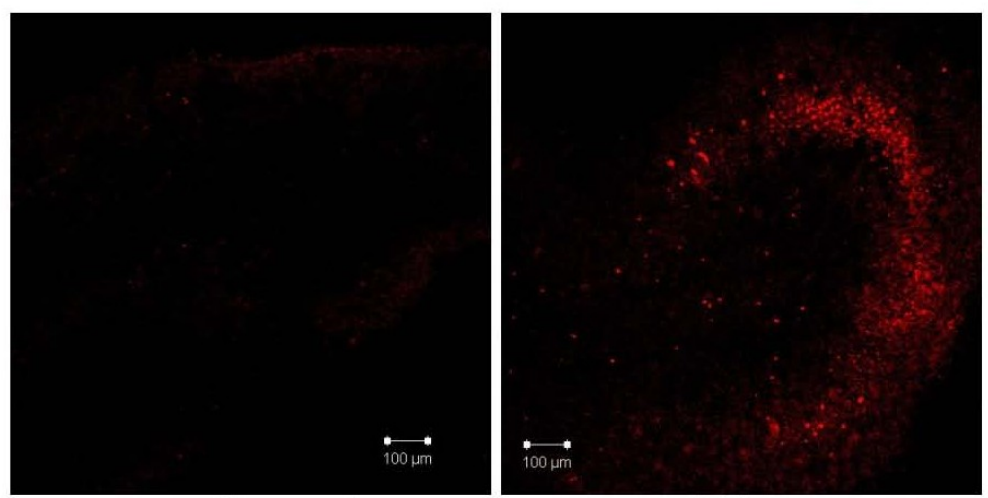

Control

OGD
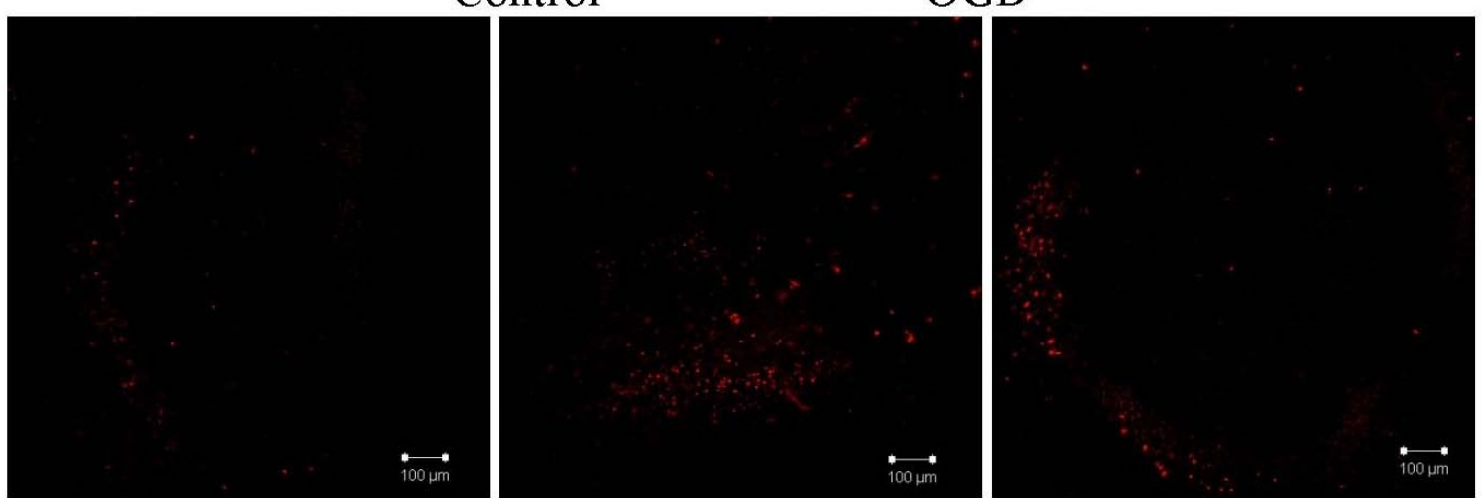

OGD + PP1

OGD + D1

OGD + D3

B

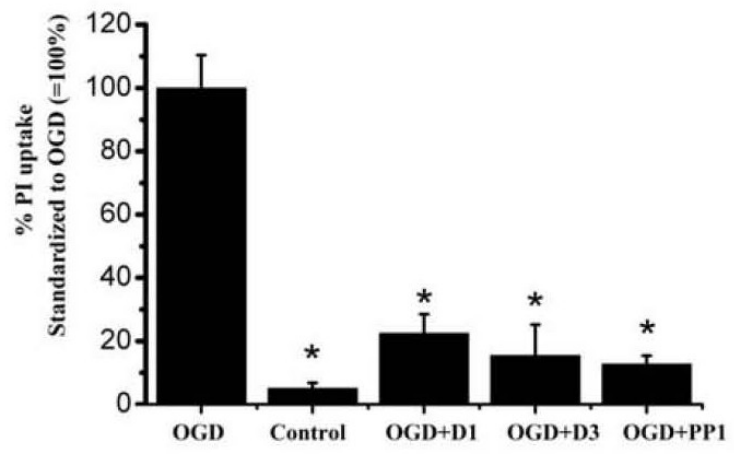




\section{Experimental Section}

\subsection{Reagents and Tested Compounds}

Dulbecco's modified Eagle's medium (DMEM), Iscove's modified DMEM (IMEM), RPMI 1640 medium, Hank's balanced solution, penicillin, streptomycin, NGF, BDNF, dimethylsulfoxide (DMSO) and the antimitotic drugs were obtained from Sigma-Aldrich; heat-inactivated fetal bovine serum was obtained from BioloT (St. Petersburg, Russia). The mouse neuroblastoma C-1300 cell line was a kind gift from Professor A.S. Holansky (Institute of Human Morphology, the Russian Academy of Medical Sciences, Moscow, Russia). Compounds 1-3 were isolated from the starfish Patiria (=Asterina) pectinifera, and compounds 4-6 were isolated from the starfish Distolasterias nipon, as described previously, and were pure according to NMR, MS, TLC, and HPLC data [13,14]. Starfish steroids were dissolved in DMSO as stock solutions ( $4 \mathrm{mM})$ and then diluted by culture media.

\subsection{NB Cell Culture}

NB C-1300 cells were grown in culture medium containing DMEM:IMEM (3:1), 5\% fetal bovine serum, $50 \mathrm{U} / \mathrm{mL}$ penicillin, and $50 \mu \mathrm{g} / \mathrm{mL}$ streptomycin at $36{ }^{\circ} \mathrm{C}$ in a $90 \%$ humidified atmosphere containing $5 \% \mathrm{CO}_{2}$. The cells were passaged every $2-3$ days.

\subsection{Neuritogenic Effects in NB Cell Cultures}

The assays for neuritogenic activity in live cells were performed according to previously described methods [11-13]. For experiments involving silver impregnation, the cells were seeded in 6-well plastic plates $\left(2 \times 10^{4}\right.$ cells per well) with glass coverslips. The cells were cultivated overnight, and the medium was replaced with that containing $2 \%$ fetal bovine serum and different concentrations of the tested compounds. DMSO in equal concentration was added to culture medium in control. At day 4 of incubation, the cultures on coverslips were fixed with bromformol for $24 \mathrm{~h}$ at room temperature and impregnated with silver according to routine protocols. The silver-impregnated preparations were assayed under a Biolar D light microscope (WZO, Poland). Digital images were used for analysis of the neuritogenic effects using the Image Tool program (Union D) according to 3 parameters: \% of cells bearing processes at least $2 \times$ the cell body diameter or bearing more than 2 processes, the number of neurites per cell, and the length of the primary neurites. In the latter case, the measured segments were summated and converted to $\mu \mathrm{m}$ using an appropriative conversion factor. A total of $>100$ cells were examined in 10 randomly chosen fields in 4 slides for each concentration of a compound, and 3 independent sets of experiments were conducted for each compound. Data are presented as the means \pm SEM. Comparisons between the control and steroid-treated groups were performed using Student's $t$-tests. A $p$-value less than 0.05 was considered statistically significant.

\subsection{Oxygen-Glucose Deprivation in NB Cell Cultures}

To induce OGD, NB cells in the logarithmic phase of growth were seeded in 24-well plates $\left(2 \times 10^{4}\right.$ cells per well in $\left.3 \mathrm{~mL}\right)$ in serum-free RPMI 1640 medium without glucose that was previously

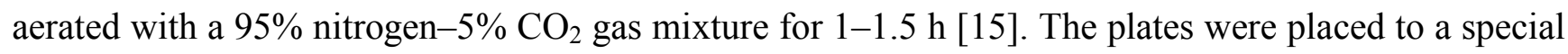


chamber aerated with the same gas mixture for $20 \mathrm{~h}$ at $36^{\circ} \mathrm{C}$ and $90 \%$ humidity. The cultures were then returned to a normoxic environment for $24 \mathrm{~h}$. Control cultures were cultivated in RPMI 1640 medium with all necessary supplements in a $90 \%$ humidified atmosphere containing $5 \% \mathrm{CO}_{2}$ at $36{ }^{\circ} \mathrm{C}$. The steroid compounds were added to the culture medium twice for the OGD period and subsequent normoxic culture period. DMSO in equal concentration was added to culture medium in control. Cell survival was assessed by adding $0.08 \%$ trypan blue dye to the culture wells for 5 min at $25{ }^{\circ} \mathrm{C}$, replacing with dye-free buffer, and counting the dye-containing (injured) and dye-excluding (viable) cells in five $40 \times$ microscope fields per well; in most cases, approximately 200 cells per well were counted. Three independent sets of experiments were conducted for each concentration of a compound. The data are reported as the means \pm SEM. Comparisons between the control and steroid-treated groups were performed using Student's $t$-tests. A $p$-value of less than 0.05 was considered statistically significant.

\subsection{Organotypic Hippocampal Slice Culture}

Organotypic hippocampal slice cultures were prepared according to previously described protocols [44]. Briefly, Wistar rats (postnatal day 7-8) were decapitated, and their brains and hippocampi were rapidly removed under aseptic conditions and placed into ice-cold Hank's balanced solution. The hippocampi were placed into agarose blocks and cut rapidly with a tissue chopper into $400 \mu \mathrm{m}$ transversal slices. The slices were transferred to collagen-coated coverslips and placed into Petri dishes containing specialized pedestals. Hank's solution was placed on the bottom of the Petri dishes to supply additional humidity. One hundred microliters of culture medium, consisting of $25 \%$ Hank's balanced solution, 65\% DMEM, and 10\% fetal bovine serum, was added to each culture. The OHSCs were transferred to a $\mathrm{CO}_{2}$-incubator and maintained in a $90 \%$ humidified atmosphere with $5 \% \mathrm{CO}_{2}$ at $36{ }^{\circ} \mathrm{C}$. At day 3 of incubation, antimitotic drugs (5-fluoro-2-deoxyuridine, cytosine- $\beta$-D-arabinofuranoside, and uridine, all from Sigma-Aldrich) were added to the culture medium to final concentrations of $1-0.1 \mu \mathrm{M}$ for $24 \mathrm{~h}$. After the removal of the antimitotics, the OHSCs were cultivated in standard culture medium (control group) or were treated with the steroid compounds at a final concentration of $1 \mu \mathrm{M}$. DMSO in equal concentration $(0.025 \%)$ was added to culture medium in control group. The medium was changed twice a week; the state of the OHSCs was controlled visually. To determine the effects of tested compounds on OHSC development after 7-10 days of cultivation, the cultures were fixed with $4 \%$ paraformaldehyde and stained with cresyl violet or impregnated with silver according to routine methods. All experimental procedures involving rats were approved by the Institutional Animal Care and Use Committee and performed according to the Directive 2010/63/EU.

\subsection{Oxygen-Glucose Deprivation Assay in OHSCs}

To analyze protective effects, the steroid compounds at the final concentration of $1 \mu \mathrm{M}$ were added to the culture medium on day 8 of cultivation. After $24 \mathrm{~h}$, the cultures were rinsed twice with RPMI 1640 medium without glucose that had been aerated previously with a $95 \%$ nitrogen- $5 \% \mathrm{CO}_{2}$ gas mixture and treated with the same medium for $35 \mathrm{~min}$ in a chamber containing $95 \%$ nitrogen $-5 \% \mathrm{CO}_{2}$. Then, the coverslips with OHSCs were rinsed twice with standard medium, placed into Petri dishes, 
and $100 \mu \mathrm{L}$ of culture medium containing the examined steroid compounds was added. The cultures were returned to the $\mathrm{CO}_{2}$-incubator. In control OHSCs, the standard culture medium was replaced with RPMI 1640 containing glucose for $35 \mathrm{~min}$ and then again replaced with standard medium. After $24 \mathrm{~h}$, the OHSCs were stained with propidium iodide (PI) $(3 \mu \mathrm{g}$ per $1 \mathrm{~mL}$ of culture medium, $60 \mathrm{~min})$, rinsed with Hank's balanced solution, fixed with 4\% paraformaldehyde $(60 \mathrm{~min}$ ) and embedded in glycerol. Finally, the OHSCs were analyzed with a LSM510 confocal laser scanning microscope (Carl Zeiss, Germany). Propidium iodide was excited with monochromatic light at $543 \mathrm{~nm}$, and images were generated using a diachronic beam splitter (FT 488/543) and a BP 585-605 nm emission bandpass filter. In order to quantify cell death, the optical density of PI fluorescence was recorded. The representative images were captured at the same exposure and digital gain settings to eliminate confounds of differential background intensity or false-positive fluorescent signal across sections. PI fluorescence was quantified using NIH ImageJ software (version 1.46). Cell death area of the $35 \mathrm{~min}$ OGD-exposed OHSCs was considered $100 \%$. Compared with this $100 \%$ value, cell death in starfish steroids-treated hippocampal slices was calculated. Statistical comparison between OGD and the starfish steroid treatments was done by oneway ANOVA. Five independent experiments were carried out. The data was expressed as a mean \pm SEM. Differences with a $p$-value $\leq 0.05$ were considered statistically significant.

\section{Conclusions}

Polar starfish steroids, analyzed in the present study, manifest neuritogenic abilities in NB cell cultures, and these effects were synergistic with the actions of NGF and BDNF. They also acted as neuroprotectors in oxygen-glucose deprivation conditions in NB cells and hippocampal slice cultures. Altogether, these results suggest that neurotrophin-like neuritogenic and neuroprotective activities are most likely common properties of starfish polyhydroxysteroids and the related glycosides, although the magnitude of these effects depends on the specific compound structures. The molecular mechanisms of these effects remain to be determined, but polar starfish steroids appear to be promising candidates for further investigation as potential neurotrophic and neuroprotective therapeutics.

\section{Acknowledgments}

The authors thank S.I. Bayborodin (Institute of Cytology and Genetics, SB RAS, Novosibirsk) for the help in experiments with confocal microscopy. The study was supported by the Program of the Presidium of RAS "Molecular and Cell Biology" (grant 12-I-P6-11), Grant No. 11-04-00052 from the RFBR, Grant No. 12-III-A-05-058 from FEB RAS, and Grant of President Russia No. 546.2012.4 supporting leading Russian scientific schools.

\section{References}

1. Bibel, M.; Barde, Y.A. Neurotrophins: Key regulators of cell fate and cell shape in the vertebrate nervous system. Genes Dev. 2000, 14, 2919-2937.

2. Huang, E.J.; Reichardt, L.F. Neurotrophins: Roles in neuronal development and function. Annu. Rev. Neurosci. 2001, 24, 677-736. 
3. Aloe, L.; Rocco, M.; Bianchi, P.; Manni, L. Nerve growth factor: From the early discoveries to the potential clinical use. J. Transl. Med. 2012, 10, 239; doi:10.1186/1479-5876-10-239.

4. Qi, J.; Luo, Y.; Gao, L. Structural diversity of neuritogenic substances and their application perspective. Mini Rev. Med. Chem. 2011, 11, 658-677.

5. More, S.V.; Koppula, S.; Kim, I.S.; Kumar, H.; Kim, B.W.; Choi, D.K. The role of bioactive compounds on the promotion of neurite outgrowth. Molecules 2012, 17, 6728-6753.

6. Ivanchina, N.V.; Kicha, A.A.; Stonik, V.A. Steroid glycosides from marine organisms. Steroids 2011, 76, 425-454.

7. Stonik, V.A.; Ivanchina, N.V.; Kicha, A.A. New polar steroids from starfish. Nat. Prod. Commun. 2008, 3, 1587-1610.

8. Dong, G.; Xu, T.H.; Yang, B.; Lin, X.P.; Zhou, X.F.; Yang, X.W.; Liu, Y.H. Chemical constituents and bioactivities of starfish. Chem. Biodivers. 2011, 8, 740-791.

9. Han, C.G.; Qi, J.H.; Ojika, M.; Linkosides, M.-Q. Neuritogenic steroid glycosides from the Okinawan starfish Linkia laevigata. J. Nat. Med. 2007, 61, 138-145.

10. Qi, J.; Han, C.; Sasayama, Y.; Nakahara, H.; Shibata, T.; Uchida, K.; Ojika, M.; Granulatoside, A. A starfish steroid glycoside, enhances PC12 cell neuritogenesis induced by nerve growth factor through an activation of MAP kinase. Chem. Med. Chem. 2006, 1, 1351-1354.

11. Pal'yanova, N.V.; Pankova, T.M.; Starostina, M.V.; Shtark, M.B.; Kicha, A.A.; Ivanchina, N.V.; Stonik, V.A. Neurotrophic effects of polyhydroxylated steroids and steroid glycosides in cultured neuroblastoma cells. Bull. Exp. Biol. Med. 2006, 141, 584-587.

12. Kicha, A.A.; Ivanchina, N.V.; Kalinovsky, A.I.; Dmitrenok, P.S.; Palyanova, N.V.; Pankova, T.M.; Starostina, M.V.; Gavagnin, M.; Stonik, V.A. New neuritogenic steroid glycosides from the Vietnamese starfish Linckia laevigata. Nat. Prod. Commun. 2007, 2, 41-46.

13. Kicha, A.A.; Kapustina, I.I.; Ivanchina, N.V.; Kalinovsky, A.I.; Dmitrenok, P.S.; Stonik, V.A.; Pal'yanova, N.V.; Pankova, T.M.; Starostina, M.V. Polyhydroxylated steroid compounds from the Far Eastern starfish Distolasterias nipon. Russ. J. Bioorg. Chem. 2008, 34, 118-124.

14. Kicha, A.A.; Ivanchina, N.V.; Gorshkova, I.A.; Ponomarenko, L.P.; Likhatskaya, G.N.; Stonik, V.A. The distribution of free sterols, polyhydroxysteroids and steroid glycosides in various body components of the starfish Patiria (=Asterina) pectinifera. Comp. Biochem. Physiol. 2001, 128B, 43-52.

15. Edsjo, A.; Holmquist, L.; Pahlman, S. Neuroblastoma as an experimental model for neuronal differentiation and hypoxia-induced tumor cell dedifferentiation. Semin. Cancer Biol. 2007, 17, 248-256.

16. Chen, P.C.; Pan, C.; Gharibani, P.M.; Prentice, H.; Wu, J.Y. Taurine exerts robust protection against hypoxia and oxygen/glucose deprivation in human neuroblastoma cell culture. Adv. Exp. Med. Biol. 2013, 775, 167-175.

17. Gulaya, N.M.; Volkov, G.L.; Klimashevsky, V.M.; Govseeva, N.N.; Melnik, A.A. Changes in lipid composition of neuroblastoma C 1300 N18 cell during differentiation. Neuroscience 1989, 30, 153-164.

18. Kostenko, M.A.; Myakisheva, S.N.; Popov, V.I. Morphological differentiation of NIE-115 mouse neuroblastoma cells. Neurosci. Behav. Physiol. 1997, 27, 516-523. 
19. Seidman, K.J.; Barsuk, J.H.; Johnson, R.F.; Weyhenmeyer, J.A. Differentiation of NG108-15 neuroblastoma cells by serum starvation or dimethyl sulfoxide results in marked differences in angiotensin II receptor subtype expression. J. Neurochem. 1996, 66, 1011-1018.

20. Clejan, S.; Dotson, R.S.; Wolf, E.W.; Corb, M.P.; Ide, C.F. Morphological differentiation of N1E-115 neuroblastoma cells by dimethyl sulfoxide activation of lipid second messengers. Exp. Cell Res. 1996, 224, 16-27.

21. Falkenstein, E.; Tillmann, H.C.; Christ, M.; Feuring, M.; Wehling, M. Multiple actions of steroid hormones-A focus on rapid, nongenomic effects. Pharmacol. Rev. 2000, 52, 513-556.

22. Numakawa, T.; Yokomaku, D.; Richards, M.; Hori, H.; Adachi, N.; Kunugi, H. Functional interactions between steroid hormones and neurotrophin BDNF. World J. Biol. Chem. 2010, 1, 133-143.

23. Fedorov, S.N.; Shubina, L.K.; Kicha, A.A.; Ivanchina, N.V.; Kwak, J.Y.; Jin, J.O.; Bode, A.M.; Dong, Z.; Stonik, V.A. Proapoptotic and anticarcinogenic activities of leviusculoside G from the starfish Henricia leviuscula and probable molecular mechanism. Nat. Prod. Commun. 2008, 3, 1575-1580.

24. Zuo, Z.; Wang, Y.; Huang, Y. Isoflurane preconditioning protects human neuroblastoma SH-SY5Y cells against in vitro simulated ischemia-reperfusion through the activation of extracellular signal-regulated kinases pathway. Eur. J. Pharmacol. 2006, 542, 84-91.

25. Ahlgren, H.; Henjum, K.; Ottersen, O.P.; Runden-Pran, E. Validation of organotypical hippocampal slice cultures as an ex vivo model of brain ischemia: Different roles of NMDA receptors in cell death signalling after exposure to NMDA or oxygen and glucose deprivation. Cell Tissue Res. 2011, 345, 329-341.

26. Cimarosti, H.; Zamin, L.L.; Frozza, R.; Nassif, M.; Horn, A.P.; Tavares, A.; Netto, C.A.; Salbego, C. Estradiol protects against oxygen and glucose deprivation in rat hippocampal organotypic cultures and activates Akt and inactivates GSK-3ß. Neurochem. Res. 2005, 30, 191-199.

27. Jin, K.L.; Mao, X.O.; Greenberg, D.A. Vascular endothelial growth factor: Direct neuroprotective effect in vitro ischemia. Proc. Natl. Acad. Sci. USA 2000, 97, 10242-10247.

28. Hedborg, F.; Ulleras, E.; Grimelius, L.; Wassberg, E.; Maxwell, P.H.; Hero, B.; Berthold, F.; Schilling, F.; Harms, D.; Sandstedt, B.; et al. Evidence for hypoxia-induced neuronal-to-chromaffin metaplasia in neuroblastoma. FASEB J. 2003, 17, 598-609.

29. Smith, I.F.; Boyle, J.P.; Vaughan, P.F.T.; Pearson, H.A.; Peers, C. Effects of chronic hypoxia on $\mathrm{Ca}(2+)$ stores and capacitative $\mathrm{Ca}(2+)$ entry in human neuroblastoma (SH-SY5Y) cells. J. Neurochem. 2001, 79, 877-884.

30. Axelson, H.; Fredlund, E.; Ovenberger, M.; Landberg, G.; Pahlman, S. Hypoxia-induced dedifferentiation of tumor cells-A mechanism behind heterogeneity and aggressiveness of solid tumors. Semin. Cell Dev. Biol. 2005, 16, 554-563.

31. Jogi, A.; Ora, I.; Nilsson, H.; Poellinger, L.; Axelson, H.; Pahlman, S. Hypoxia-induced dedifferentiation in neuroblastoma cells. Cancer Lett. 2003, 197, 145-150.

32. Pahlman, S.; Stockhausen, M.T.; Fredlund, E.; Axelson, H. Notch signaling in neuroblastoma. Semin. Cancer Biol. 2004, 14, 365-373. 
33. Jogi, A.; Vallon-Christersson, J.; Holmquist, L.; Axelson, H.; Borg, A.; Pahlman, S. Human neuroblastoma cells exposed to hypoxia: Induction of genes associated with growth, survival, and aggressive behavior. Exp. Cell Res. 2004, 295, 469-487.

34. Holmquist, L.; Lofstedt, T.; Pahlman, S. Effect of hypoxia on the tumor phenotype: The neuroblastoma and breast cancer models. Adv. Exp. Med. Biol. 2006, 587, 179-193.

35. Nilsson, H.; Jogi, A.; Beckman, S.; Harris, A.L.; Poellinger, L.; Pahlman, S. HIF-2 $\alpha$ expression in human fetal paraganglia and neuroblastoma: Relation to sympathetic differentiation, glucose deficiency, and hypoxia. Exp. Cell Res. 2005, 303, 447-456.

36. Hoehner, J.C.; Prabhakaran, K. Induced differentiation affords neuroblastoma cells protection from hypoxic injury. J. Pediatr. Surg. 2003, 38, 1069-1074.

37. Gerace, E.; Landucci, E.; Scartabelli, T.; Moroni, F.; Pellegrini-Giampietro, D.E. Rat hippocampal slice culture models for the evaluation of neuroprotective agents. Methods Mol. Biol. 2012, 846, 343-354.

38. Radley, E.; Akram, A.; Grubb, B.D.; Gibson, C.L. Investigation of the mechanisms of progesterone protection following oxygen-glucose deprivation in organotypic hippocampal slice cultures. Neurosci. Lett. 2012, 506, 131-135.

39. Zamin, L.L.; Dillenburg-Pilla, P.; Argenta-Comiran, R.; Horn, A.P.; Simão, F.; Nassif, M.; Gerhardt, D.; Frozza, R.L.; Salbego, C. Protective effect of resveratrol against oxygen-glucose deprivation in organotypic hippocampal slice cultures: Involvement of PI3-K pathway. Neurobiol. Dis. 2006, 24, 170-182.

40. Green, P.S.; Simpkins, J.W. Neuroprotective effects of estrogens: Potential mechanisms of action. Int. J. Dev. Neurosci. 2000, 18, 347-358.

41. Zhao, L.Q.; Brinton, R.D. Select estrogens within the complex formulation of conjugated equine estrogens $\left(\right.$ Premarin $^{\circledR}$ ) are protective against neurodegenerative insults: implications for a composition of estrogen therapy to promote neuronal function and prevent Alzheimer's disease. BMC Neurosci. 2006, 7, 24-36.

42. Hurn, P.D.; Macrae, I.M. Estrogen as a neuroprotectant in stroke. J. Cereb. Blood Flow Metab. 2000, 20, 631-652.

43. Singh, M.; Su, C. Progesterone, brain-derived neurotrophic factor and neuroprotection. Neuroscience 2012, 239, 84-91.

44. Gahwiler, B.H.; Capogna, M.; Debanne, D.; McKinney, R.A.; Thompson, S.M. Organotypic slice cultures: A technique has come of age. Trends Neurosci. 1997, 20, 471-477.

(C) 2013 by the authors; licensee MDPI, Basel, Switzerland. This article is an open access article distributed under the terms and conditions of the Creative Commons Attribution license (http://creativecommons.org/licenses/by/3.0/). 